\title{
Asymmetric Dependence Patterns in Financial Time Series
}

\author{
Manuel Ammann and Stephan Süss ${ }^{\mathrm{a}}$
}

December 3, 2008

\begin{abstract}
This paper proposes a new copula-based approach to test for asymmetries in the dependence structure of financial time series. Simply splitting observations into subsamples and comparing conditional correlations leads to spurious results due to the well-known conditioning bias. Our suggested framework is able to circumvent these problems. Applying our test to market data, we statistically confirm the widespread notion of significant asymmetric dependence structures between daily changes of the VIX, VXN, VDAXnew, and VSTOXX volatility indices and their corresponding equity index returns. A maximum likelihood method is used to perform a likelihood ratio test between the ordinary t-copula and its asymmetric extension. To the best of our knowledge, our study is the first empirical implementation of the skewed t-copula to generate meta skewed student t-distributions. Its asymmetry leads to significant improvements in the description of the dependence structure between equity returns and implied volatility changes.
\end{abstract}

JEL Classification: C01, Key Words: Copulae, Asymmetric Dependence Concepts

${ }^{a}$ Manuel Ammann is Professor of Finance at the Swiss Institute of Banking and Finance, University of St. Gallen, Switzerland.

Stephan Süss is research assistant at the Swiss Institute of Banking and Finance, University of St. Gallen, Switzerland.

Address for Correspondence: Swiss Institute for Banking and Finance, University of St. Gallen, Rosenbergstrasse 52, CH-9000 St. Gallen, Switzerland. Voice: +41 7122470 13, Email: stephan.suess@unisg.ch

We thank the participants of the "Conference on Copulae and Multi-Variate Probability Distributions in Finance" in Warwick, UK, and our anonymous referees for very helpful suggestions and comments. 


\section{Introduction}

This study uses copula theory to construct a likelihood ratio based test for asymmetric dependence structures. As an application, we confirm the well-known empirical property of rising dependence between daily changes of implied volatility and equity market returns during periods of financial market turmoil. This is shown for CBOE's implied volatility index VIX, the VXN, Germany's VDAXnew, and the VSTOXX and daily returns of their corresponding equity indices. The dynamics of option implied volatility has been widely studied (see e.g. Fleming, Ostdiek, and Whaley (1995), Moraux, Navatte, and Villa (1998), Whaley (2000), Blair, Poon, and Taylor (2001), Simon (2003)), and Corrado and Miller (2005). One of the main findings is that in periods of heightened equity market volatility, correlations between returns and implied volatility changes increase in magnitude. The usually applied approach to test for unstable correlations is to condition the estimated correlation coefficient on ex post realizations of equity market returns. An implementation of this research design comprises several drawbacks, however. First, Boyer, Gibson, and Loretan (1999), Longin and Solnik (2001), Forbes and Rigobon (2002), as well as Ang and Chen (2002) note that conditional correlation is highly nonlinear in the volatility of the returns on which it is conditioned. This finding became known as the "conditioning bias". For illustration purposes, consider the simple introductory example of Boyer, Gibson, and Loretan (1999): Let $x$ and $y$ be two normally distributed univariate random variables with means $\mu_{x}$ and $\mu_{y}$, respectively, standard deviations of $\sigma_{x}$ and $\sigma_{y}$, and a correlation coefficient of $\rho_{x y}\left(=\sigma_{x y} / \sigma_{x} \sigma_{y}\right)$. Using a Cholesky decomposition, these can be written as

$$
\begin{aligned}
& x=\mu_{x}+\sigma_{x} u \\
& y=\mu_{y}+\rho_{x y} \sigma_{y} u+\sqrt{1-\rho_{x y}} \sigma_{y} \nu
\end{aligned}
$$


where $u, \nu \sim N(0,1)$. Now consider an event $x \in \mathcal{A}$ for any $\mathcal{A} \subset \mathbb{R}$ with $0<\operatorname{Prob}(\mathcal{A})<1$. The conditional correlation coefficient is now calculated as

$$
\rho_{\mathcal{A}}=\frac{\operatorname{Cov}(x, y \mid x \in \mathcal{A})}{\sqrt{\operatorname{Var}(x \mid x \in \mathcal{A})} \sqrt{\operatorname{Var}(y \mid x \in \mathcal{A})}} .
$$

Inserting (1) and rearranging leads to

$$
\rho_{\mathcal{A}}=\rho_{x y}\left[\rho_{x y}^{2}+\left(1-\rho_{x y}^{2}\right) \frac{\sigma_{x}^{2}}{\operatorname{Var}(x \mid x \in \mathcal{A})}\right]^{-1 / 2} .
$$

As such, simply splitting samples of ex post return realizations can yield to misleading results, due to the dependence of $\rho_{\mathcal{A}}$ on $\operatorname{Var}(x \mid x \in \mathcal{A})$.

Second, the empirical estimation of the conditional correlations causes serious biases. Its results are severely dependent upon the point of the sample split. With the cut-off frontier moving further into the tails of the distribution, fewer observations are included in the estimation and the weight of single outliers might distort the estimation. Conversely, moving the cut-off frontier towards the core of the distribution sharply increases the observation number. Yet, the correlation coefficient is now dominated by the bulk of the observations in the center and is unable to capture the behavior of the dependence pattern in the tails.

Finally, it is a well-known fact that empirical distributions of financial market variables such as equity returns or interest rates typically exhibit strong nonGaussian features. They are usually leptokurtic and skewed with one heavy and one Gaussian-like tail. ${ }^{1}$ Using correlation coefficients to measure dependence is only appropriate when the joint distribution of the time series is multivariate normal and therefore cannot properly account for these patterns. ${ }^{2}$ Several authors have proposed methods to circumvent these problems. ${ }^{3}$ Ang and Bekaert (2002), for example, implement an asymmetric GARCH model with time-varying volatilities and constant correlation. They conclude that these specifications cannot

\footnotetext{
${ }^{1}$ See, e.g. Aas and Haff (2006).

${ }^{2}$ See, e.g. Embrechts, McNeil, and Straumann (2002).

${ }^{3}$ See, e.g. Koutmos and Booth (1995), Fortin and Kusmics (2002), and the references therein.
} 
properly account for the asymmetry in the dependence structure. Longin and Solnik (2001) use multivariate extreme value theory. Ang and Bekaert (2002), and Das and Uppal (2004) implement regime-switching models for modeling timevarying dependence patterns in the time series. All of these studies reject symmetric correlation patterns in equity return time series.

The first contribution of this paper is a formal test for asymmetric dependence patterns in multivariate distributions. As copulas isolate the dependence pattern from the marginal distributions, we are able to alleviate the biases deriving from the conditioning of observations. An attractive feature of our methodology is that it is not specific to any model of the marginal time series processes and therefore to the marginal distributions. The idea is simple: We fit a skewed t-copula by pseudo maximum likelihood independently of the univariate marginal time series models. ${ }^{4}$ Setting all its skewness parameters equal to zero results in the ordinary t-copula as a special case. A pseudo likelihood ratio test between the skewed t-copula and its symmetric counterpart therefore provides a simple tool to test for asymmetric dependence structures.

The specification of the skewed student t-distribution we apply in our framework is mentioned by Prause (1999), Barndorff-Nielsen and Shephard (2001), Jones and Faddy (2003), and Menca and Sentana (2004). Little, however, is known about its behavior in practical situations. Aas and Haff (2006), and Hu (2006) outline its first empirical implementations for risk management applications. Demarta and McNeil (2005) derive its copula. To the best of our knowledge, our study constitutes the first implementation of its copula to generate meta skewed student t-distributions. The estimation procedure is based on an augmented version of the EM algorithm outlined in Demarta and McNeil (2005), and McNeil,

\footnotetext{
${ }^{4}$ The likelihood is called "pseudo" as it is conditioned on the time series models of the univariate marginal distributions. Genest, Ghoudi, and Rivest (1995) prove the consistency and asymptotic normality of the copula parameter estimators if the data sample is iid. Also see, among others, Demarta and McNeil (2005).
} 
Frey, and Embrechts (2005).

The empirical contribution of this paper is to apply our framework to test for asymmetric dependence patterns in the dynamics between the VIX, VXN, VDAXnew, and VSTOXX implied volatility changes and the returns of their corresponding equity indices. Our concept is related to GARCH type models with asymmetric conditional volatility distributions. These specifications have become popular in the literature during the past decade. The connection to our setting can intuitively be seen in equation (3). Introducing skewness increases the conditional variance in the tails. This in turn leads to higher absolute conditional correlation values. The main disadvantage of these settings is the close connection between the shapes of the univariate distributions and the dependence structure. Using copula theory, we are able to separate the variance distribution from the dependence structure.

Our findings strongly confirm the general impression of rising negative dependence between both time series during equity market turmoils.

The paper is organized as follows: Section 2 outlines the construction principle of our test for asymmetric dependence structures. The subsequent Section 3 provides an empirical implementation to test for rising dependence between daily S\&P 500 equity returns and VIX level changes. Section 4 concludes. The EM algorithm to estimate parameters of the skewed t-copula is illustrated in the Appendix.

\section{A Model to Test for Asymmetric Conditional Dependence}

This section develops a copula-based framework to test for asymmetry in the dependence structure between two random variables. For excellent introductions into this topic, we recommend Joe (1997), Nelsen (1999), and Embrechts, McNeil, 
and Straumann (2002).

Our suggested setting is similar to the bivariate GARCH models of Patton (2006), Jondeau and Rockinger (2001), and $\mathrm{Hu}$ (2006). It is most related to that of Fortin and Kusmics (2002), who found co-movements of extreme stock return pairs.

We fit parametric copula families using a two-stage procedure, as proposed in Oakes (1994), Genest, Ghoudi, and Rivest (1995), and Shih and Louis (1995). This approach comprises the transformation of univariate time series into uniformly distributed vectors. Then, the dependence parameters are estimated by the maximization of a pseudo log-likelihood function.

The general idea of the test does not depend on a specific model for the univariate marginal processes. For ease of exposition, we estimate auto-regressive $\operatorname{GARCH}(1,1)$ models for the time series of equity index returns, and volatility index level changes. Consider a $d$-dimensional time series sample of length $T$. Specify for each univariate time series $j \in\{1, \ldots, d\}$ the model

$$
\begin{aligned}
& x_{j, t}=\alpha+\sum_{k=1}^{K_{j}} \phi_{k} x_{j, t-k}+\sigma_{j, t} \tilde{\varepsilon}_{j, t} \\
& \sigma_{j, t}^{2}=\omega_{j}+\beta_{j} \varepsilon_{j, t-1}^{2}+\gamma \sigma_{j, t-1}^{2},
\end{aligned}
$$

where $\tilde{\varepsilon}_{j, t}$ follows an iid student t-distribution with $\nu_{j}$ degrees of freedom, i.e. $\varepsilon \sim t_{\nu_{j}}(0,1) . K_{j}$ is determined by optimizing the Bayesian Information Criterion (BIC). Using the probability integral transform we can infer for the $j$-th marginal distribution $\hat{u}_{j, t}=\hat{F}_{\nu_{j}}^{t}\left(x_{j, t}\right)$, where $F_{\nu}^{t}$ denotes the univariate t-distribution with $\nu$ degrees of freedom. Forming the vectors $\hat{\mathbf{u}}_{t}=\left(\hat{F}_{t, 1}, \ldots, \hat{F}_{t, d}\right)^{\prime}, \forall t=1, \ldots, T$ allows to estimate the considered copula parameters by maximizing the pseudo log-likelihood function

$$
\ln \mathscr{L}=\sum_{t=1}^{T} \ln c\left(\hat{\mathbf{u}}_{1}, \ldots, \hat{\mathbf{u}}_{d}, \theta\right),
$$

where $c\left(\hat{\mathbf{u}}_{1}, \ldots, \hat{\mathbf{u}}_{d}\right)=\frac{\partial C^{d}\left(\hat{\mathbf{u}}_{1}, \ldots, \hat{\mathbf{u}}_{d}\right)}{\partial \hat{\mathbf{u}}_{1} \cdots \partial \hat{\mathbf{u}}_{d}}$ denotes the copula density and $\theta$ the collection of parameters required to determine the dependence structure. 
As already mentioned, our study applies the student t and the skewed t-copula. For a brief outline of both dependence patterns, we consider their mixture representations.

$\mathbf{X}=\left(t_{\nu}^{-1}\left(u_{1}\right), \ldots, t_{\nu}^{-1}\left(u_{d}\right)\right)^{\prime}$ is said to follow a t-distribution with $\nu$ degrees of freedom, if

$$
\mathbf{X} \sim \mu+\sqrt{W} \mathbf{Z}
$$

where $\mathbf{Z} \sim N(\mathbf{0}, \boldsymbol{\Sigma})$, and $W$ is independent of $\mathbf{Z}$, satisfying $\nu / W \sim \chi_{\nu}^{2}$. The d-dimensional t-distribution can be written as

$$
\begin{aligned}
& F_{\nu, \boldsymbol{\Sigma}}^{t}\left(x_{1}, \ldots, x_{d}\right)= \\
& \int_{-\infty}^{x_{1}} \cdots \int_{-\infty}^{x_{d}} \frac{\Gamma\left(\frac{\nu+d}{2}\right)}{\Gamma\left(\frac{\nu}{2}\right) \sqrt{(\pi \nu)^{d}|\mathbf{\Sigma}|}}\left(1+\frac{(\mathbf{X}-\mu)^{\prime} \boldsymbol{\Sigma}^{-1}(\mathbf{X}-\mu)}{\nu}\right)^{-\frac{\nu+d}{2}} d x_{1} \ldots d x_{d},
\end{aligned}
$$

where $\Gamma$ denotes Euler's Gamma Function.

A random vector $\mathbf{X}$ has a skewed $t$ distribution if

$$
\mathbf{X} \sim \mu+\gamma W+\sqrt{W} \mathbf{Z}
$$

where $\mathbf{Z} \sim N(\mathbf{0}, \boldsymbol{\Sigma})$, and $\mathrm{W}$ has the inverse gamma distribution $I(\nu / 2, \cdots, \nu / 2)$. The corresponding distribution function can be written as

$$
F_{\nu, \boldsymbol{\Sigma}, \gamma}^{t}\left(x_{1}, \ldots, x_{d}\right)=\int_{-\infty}^{x_{1}} \cdots \int_{-\infty}^{x_{d}} f_{\nu, \boldsymbol{\Sigma}, \gamma}^{t}(\mathbf{X}) d x_{1} \ldots d x_{d}
$$

where

$$
f_{\nu, \boldsymbol{\Sigma}, \gamma}^{t}(\mathbf{X})=c \frac{K_{\frac{\nu+d}{2}}\left(\sqrt{\left(\nu+(\mathbf{X}-\mu)^{\prime} \boldsymbol{\Sigma}^{-1}(\mathbf{X}-\mu)\right) \gamma^{\prime} \boldsymbol{\Sigma}^{-1} \gamma}\right) \exp \left((\mathbf{X}-\mu)^{\prime} \boldsymbol{\Sigma}^{-1} \gamma\right)}{\left(\sqrt{\left(\nu+(\mathbf{X}-\mu)^{\prime} \boldsymbol{\Sigma}^{-1}(\mathbf{X}-\mu)\right) \gamma^{\prime} \boldsymbol{\Sigma}^{-1} \gamma}\right)^{-\frac{\nu+d}{2}}\left(1+\frac{(\mathbf{X}-\mu)^{\prime} \boldsymbol{\Sigma}^{-1}(\mathbf{X}-\mu)}{\nu}\right)^{\frac{\nu+d}{d}}} .
$$

$K_{\lambda}$ denotes the modified Bessel function of the third kind. The normalizing constant $c$ is defined as

$$
c=\frac{2^{\frac{2-(\nu+d)}{2}}}{\Gamma\left(\frac{\nu}{2}\right)(\pi \nu)^{\frac{d}{2}}|\Sigma|^{\frac{1}{2}}} .
$$

Due to the strict monotonicity of the (skewed) t-distribution, we can infer the d-dimensional copula function applying Sklar's Theorem by

$$
C_{\nu, \boldsymbol{\Sigma},(\gamma)}^{t}\left(\hat{u}_{1, t}, \ldots, \hat{u}_{d, t}\right)=F_{\nu, \boldsymbol{\Sigma},(\gamma)}^{t}\left(\left(\hat{F}_{\nu}^{t}\left(\hat{u}_{1, t}\right)\right)^{-1}, \ldots,\left(\hat{F}_{\nu}^{t}\left(\hat{u}_{d, t}\right)\right)^{-1}\right),
$$


where $\left(\hat{F}_{\nu}^{t}\left(\hat{u}_{j, t}\right)\right)^{-1}$ denotes the inverse student $\mathrm{t}$ distribution function. Note that (8) nests the student $t$ dependence structure as $\gamma \rightarrow \mathbf{0}$. The Gaussian case is obtained by additionally letting $\nu \rightarrow \infty$.

With this setting we can control for tail dependence by altering the degrees of freedom. Furthermore, we are able to account for asymmetries in the dependence structure by varying the skewness vector $\gamma$. A simple "pseudo likelihood ratio test" between the ordinary t-copula and its generalization is able to test for the significance of these asymmetries.

\section{The Asymmetric Dependence Structure Be- tween Volatility Index Level Changes and Eq- uity Index Returns}

There has been a large amount of empirical research investigating the rising return dependence of different equity markets during periods of financial market turmoil. This section highlights a comparable, yet negative, relationship between volatility indices and their "underlying" equity indices which was first mentioned by Whaley $(2000)$.

\subsection{Data}

Data for this study are distributed over several time periods and equity markets. Our observations of daily VIX closing levels and returns of its corresponding S\&P 500 index spread from January $2^{\text {nd }}$, 1990, to September $29^{\text {th }}$, 2007. Although the dissemination of the new VIX calculation took place on September $22^{\text {nd }}$, 2003, daily historical values are available since the beginning of $1990 .^{5}$ After the

\footnotetext{
${ }^{5}$ Values of the new VIX have been backfilled to January $2^{\text {nd }}$, 1990. As its calculation is only based on observable values, it can be determined in an unbiased manner. For its theoretic
} 
exclusion of non-trading days, our sample contains a total of 4475 closing index values for both time series.

The high success of the VIX as an accepted measure of implied market volatility led to the introduction of several different volatility indices. On February $2^{\text {nd }}$, 2001, CBOE additionally launched VXN, tracking implied volatility figures of Nasdaq 100 equity index options. Consideration of the entire history of daily closing levels and Nasdaq 100 returns leads to sample lengths of 1672 observations each.

On December, $5^{\text {th }}$, 1994, the German Futures and Options Exchange (DTB) launched VDAX, currently termed VDAXnew after a change in its calculation method. It tracks implied volatility of DAX index options. Our study includes daily observations of DAX and VDAXnew closing levels for the January $2^{\text {nd }}, 1992$, to September $29^{t h}, 2007$, period. ${ }^{6}$ The exclusion of non-trading days leads to a total of 3983 observations for each time series.

Finally, we investigate the European implied volatility index VSTOXX that was introduced on January $4^{\text {th }}, 1999$. It tracks the implied volatility levels of DJ Euro STOXX 50 index options. Daily equity index log-returns and closing levels have series lengths of 2233 observations.

Figure 1 illustrates scatter plots of the four samples. The strong negative linear dependence with unconditional correlation coefficients of $\rho\left(r_{S \& P 500}, \Delta V I X\right)=$ $-0.7374, \rho\left(r_{\text {Nasdaq100 }}, \Delta V X N\right)=-0.6063, \rho\left(r_{D A X}, \Delta V D A X n e w\right)=-0.6615$, and $\rho\left(r_{D J E u r o S T O X X 50}, \Delta V S T O X X\right)=-0.7650$ is clearly observable in each graph. This is in line with the results first mentioned by Whaley (1993). High equity returns are typically accompanied by negative changes in volatility index levels, while huge upward spikes of the latter are most likely observable during construction, see e.g. Carr and Madan (1998), Demeterfi, Derman, Kamal, and Zou (1999), and Britten-Jones and Neuberger (2000).

${ }^{6}$ Using a similar calculation method to the VIX, values of the VDAXnew have been backfilled to January $2^{\text {nd }}, 1992$. 
equity market downturns. The clustering of observations is less tight in the tails than in the core of the distribution. Therefore, calculated correlation coefficients might be distorted, especially due to positive outliers of volatility index level changes.

\section{[Please insert Figure 1 around here]}

Daily volatility index closing levels and the corresponding equity log-returns are displayed in Figure 2. In Figure (a), CBOE's VIX reveals several periods of relatively high levels, usually initiated by a sharp upward spike in connection with large squared S\&P 500 log-returns. These include the period of the first Gulf War from mid 1990 until the beginning of 1991, the Asian currency crises in 1997, the period of the Russian restructuring starting in August 1998 in combination with the restructuring of LTCM shortly thereafter, the successive bursting of the dotcom bubble in 2000 to 2001, the terrorist attack on September 11 ${ }^{\text {th }}, 2001$ and the period of the American invasion of Iraq in March 2003. Similar patterns can be seen in the behavior of the other three sample time series. Typically, high market returns are accompanied by negative changes in implied volatility levels, while pronounced equity market downturns lead to huge upward spikes in volatility.

\section{[Please insert Figure 2 around here]}

Descriptive statistics of all sample time series are given in Table 1. Regarding equity index log-returns, we find mean and median values to be of very small magnitude. Skewness parameters are typically negative, ranging from - 0.085 for

the DJ Euro STOXX 50 to -0.252 for DAX returns. An exemption from this finding is the positive skewness of 0.105 for Nasdaq 100 returns during our sample 
period. Jarque-Bera and augmented Dickey-Fuller tests can be rejected for all four time series to a significance level of one per cent.

\section{[Please insert Table 1 around here]}

Closing levels of implied volatility indices have averages ranging from 18.933 for VIX levels to 29.695 for VXN levels. The distributions typically have a positive skewness, reflecting the existence of positive outliers which can also be detected in the time series plots of Figure 2. Jarque-Bera tests can be rejected for all four time series to a level of one per cent.

Our study uses implied volatility level changes rather than closing index values. It is well-known in the literature that implied volatility indices are highly persistent and hence have a low degree of mean-reversion. ${ }^{7}$ The characteristic polynomial of their processes typically has a root close to unity. Therefore, their processes are frequently referred to be "near-integrated". This is confirmed by our sample: Table 1 outlines the statistics of augmented Dickey-Fuller tests for implied volatility index closing levels. Although they can be rejected for the VIX and DAX series to a level of one per cent, the statistics are very close to the $1 \%$ critical values. The corresponding test for VXN and VSTOXX closing levels cannot be rejected with p-value of 0.203 and 0.018 , respectively. Therefore, we use implied volatility level changes for our study rather than closing levels. ${ }^{8}$ Descriptive statistics for these time series are also outlined in Table 1. Difference means and medians have values around zero for all four time series. Daily VXN level changes show the lowest average value of -0.020 . This finding can mainly be attributed to the negative index trend during the short sample observation period. This can also

\footnotetext{
${ }^{7}$ See, e.g. Fleming, Ostdiek, and Whaley (1995), Moraux, Navatte, and Villa (1998), Whaley (2000), Blair, Poon, and Taylor (2001), Corrado and Miller (2005), as well as Simon (2003). This list is by no means exhaustive.

${ }^{8} \mathrm{~A}$ different method would be to use changes in the logarithm of the closing level. This method leads to conclusions which are very similar to our results.
} 
be seen in Figure 2. All four series have a positive skewness, ranging between 0.270 for the VXN to 1.304 for the VSTOXX. Jarque-Bera tests for normality and augmented Dickey-Fuller tests for the presence of unit roots can convincingly be rejected for all four time series.

\subsection{Estimating the Asymmetry in the Dependence Pat- tern}

Before we turn to the implementation of our copula test, we estimate the correlation structure of return exceedances. These are defined with cut-off frontiers $\vartheta$. Subsamples for the calculation of conditional correlations include all observations $\mathbf{X}_{\vartheta}$ with $r_{\text {EquityIndex }}\left(\mathbf{X}_{\vartheta}\right) \in(-\infty, \vartheta], \forall \vartheta \leq 0$ and $r_{\text {EquityIndex }}\left(\mathbf{X}_{\vartheta}\right) \in[\vartheta, \infty), \forall \vartheta>$ 0, respectively. The estimated values are illustrated in Figure 3.

\section{[Please insert Figure 3 around here]}

The four time series pairs display very similar patterns. Correlation coefficients are severely dependent upon $\vartheta$. In addition, as can be seen for high absolute equity return values, correlations become more erratic with higher absolute values of $\vartheta$. This is mainly due to outliers distorting calculations. The structure of return exceedance correlations is typically asymmetric. Moving into the distribution tails, $\rho_{\vartheta}$ rises in a steeper manner for positive equity returns. This seems counterintuitive at first sight. The positive skewness of volatility index changes in combination with negative third moments of equity returns should lead to higher conditional variances and therefore to faster reductions of absolute correlation figures for negative values of $\vartheta$. Yet, the behavior of correlation coefficients for negative equity returns can be explained by the existence of positive outliers in volatility changes. They lead to the calculation of distorted and very negative correlation coefficients. Regarding these aspects, one cannot gauge the magnitude 
of asymmetric dependence patterns by simply comparing empirical conditional correlations.

In order to circumvent these problems we implement the copula-based test for dependence asymmetries outlined in the previous section. The estimated parameters of the marginal time series models introduced in (4) are provided in Table 2.

\section{[Please insert Table 2 around here]}

Figure 4 illustrates the empirical joint distribution mapped into the unit square by inverting the residuals of (4). The high concentration of concurrent extreme values is clearly observable for all four time series pairs. This is especially true for the upper-left tails.

\section{[Please insert Figure 4 around here]}

Tail dependence, although a widespread concept, is an asymptotic quantity. Therefore, we calculate the empirical ratios

$$
\frac{\operatorname{Prob}\left(r_{\text {EquityIndex }} \leq u, \Delta \text { Volatility Index } \geq 1-u\right)}{u},
$$

for $u=0.05,0.1$, and 0.2 . For tackling the dependence structure in the lower-right tail, we analogously determine

$$
\frac{\operatorname{Prob}\left(r_{\text {EquityIndex }} \geq \bar{u}, \Delta \text { Volatility Index } \leq 1-\bar{u}\right)}{1-\bar{u}},
$$

where $\bar{u}=0.8,0.9$, and 0.95 . These can be interpreted as "empirical joint conditional quantile exceedance probabilities". ${ }^{9}$ They are outlined in Table 3.

\footnotetext{
${ }^{9}$ See, e.g. Demarta and McNeil (2005).
} 


\section{[Please insert Table 3 around here]}

Empirical quantile exceedance probabilities of the upper-left tail are up to 7.1 times higher than their corresponding lower-right values. The magnitudes of the differences are stated in brackets. In order to gauge their significance, we fit the student t-copula and its skewed generalization. We then perform a likelihood ratio test between both dependence patterns as outlined in the previous section. Obtained results are presented in Table 4.

\section{[Please insert Table 4 around here]}

The high concentration of observations in the tails which is also shown in Figure 4 translates into very low degrees of freedom values ranging between 3.06 and 5.59 for the student t-copula and between 6.30 and 7.24 for its asymmetric counterpart. $\nu$ is slightly lower for the student t-copula for all four time series pairs. This might be due to the fact that the dependence structure of the skewed t-distribution is able to capture the high concentration of observations in the upper-left tail by the skewness parameters rather than by lower degrees of freedom. $\gamma$ is typically negative for equity return time series and positive for volatility index level changes. This results in high dependence values in the upper-left tails of the bivariate distributions. One exemption from this finding is the slightly positive value of 0.008 for the Nasdaq 100 time series. This finding might be attributed to its short sample period which contains only very few abrupt equity market downturns which are accompanied by positive upward spikes in the corresponding volatility index VXN. (See also the time series plot in Figure 2.) Log-likelihood values are range between -10322.52 $\left(r_{S \& P 500} / \Delta V I X\right)$ and -4934.82 $\left(r_{\text {Nasdaq100 }} / \Delta V X N\right)$ for the symmetric setting and between -10047.67 $\left(r_{S \& P 500} / \Delta V I X\right)$ and -4282.98 $\left(r_{\text {Nasdaq100 }} / \Delta V X N\right)$ for its generalized framework. Pseudo likelihood ratio tests can be rejected with a significance level of 1 per cent for all four time series pairs. 
This is confirmed by the information criteria of Akaike and Schwarz. Both lead to the rejection of the student t-copula in favor of its skewed generalization for all four dependence structures.

We conclude that the dependence between equity index log-returns and implied volatility level changes is significantly higher in case of market downturns.

\section{Conclusion}

We use copula theory to test for asymmetric dependence structures between daily changes of the VIX, VXN, VDAXnew, and VSTOXX volatility index changes and their corresponding equity index returns. As a first contribution, we propose a likelihood ratio test that is able to control for both tail dependence and asymmetries in dependence structures. The idea is simple and easy to implement. Conditional on an ordinary AR-GARCH model for the univariate marginal distributions, we fit a skewed t-copula as outlined in Demarta and McNeil (2005). As this dependence structure entails the ordinary t-copula as a special case, a simple likelihood ratio test serves to test for asymmetry in the dependence pattern of the joint distribution.

Applying our framework to empirical data, we find that the negative dependence between daily equity returns and level changes of implied volatility significantly increases during periods of equity market turmoil. To the best of our knowledge, this study is the first empirical implementation of the skewed t-copula to generate meta skewed t-distributions. Our estimation is based on a slightly modified version of the EM algorithm by Demarta and McNeil (2005). Its application leads to significantly better empirical fits in the description of the dependence pattern between equity returns and implied volatility changes. 


\section{References}

Aas, K., And I. HafF (2006): "The Generalized Hyperbolic Skew Student's t-distribution," The Journal of Financial Econometrics, 4(2), 275-309.

Ang, A., And G. Bekaert (2002): "International Asset Allocation With Regime Shifts," The Review of Financial Studies, 15(4), 1137-1187.

Ang, A., And J. Chen (2002): "Asymmetric Correlations of Equity Portfolios," The Journal of Financial Economics, 63(3), 443-494.

Barndorff-Nielsen, O., And N. Shephard (2001): "Normal Modified Stable Processes," Theory of Probability and Mathematical Statistics, 65(1), 1-19.

Blair, B., S.-H. Poon, and S. TAYlor (2001): "Forecasting S\&P 100 Volatility: The Incremental Information Content of Implied Volatility and HighFrequency Index Returns," The Journal of Econometrics, 105(1), 2-26.

Boyer, B., M. Gibson, and M. Loretan (1999): "Pitfalls in Tests for Changes in Correlations," Board of Governors of the Federal Reserve System: International Finance Discussion Papers, Number $59 \%$.

Britten-Jones, M., And A. Neuberger (2000): "Option Prices, Implied Price Processes, and Stochastic Volatility," The Journal of Finance, 55(2), $839-866$.

Carr, P., And D. Madan (1998): Towards a Theory of Volatility Trading, in: Volatility: New Estimation Techniques for Pricing Derivatives chap. 29, pp. 417-427. Risk Publications.

Corrado, C., And T. Miller (2005): "The Forecast Quality of CBOE Implied Volatility Indexes," The Journal of Futures Markets, 25(4), 339-373.

Das, S., And R. UpPal (2004): "Systemic Risk and International Portfolio Choice," The Journal of Finance, 59(6), 2809-2834. 
Demarta, S., And A. MCNeil (2005): "The t Copula and Related Copulas," International Statistical Review, 73(1), 111-129.

Demeterfi, K., E. Derman, M. Kamal, and J. Zou (1999): "A Guide to Volatility and Variance Swaps," The Journal of Derivatives, 6(4), 9-32.

Embrechts, P., A. McNeil, and D. Straumann (2002): Correlation and Dependence in Risk Management: Properties and Pitfalls, In: Risk Management: Value at Risk and Beyond pp. 176-223. Cambridge University Press, Cambridge.

Fleming, J., B. Ostdiek, and R. Whaley (1995): "Predicting Stock Market Volatility: A New Measure," The Journal of Futures Markets, 15(3), 265-302.

Forbes, K., and R. Rigobon (2002): "No Contagion, Only Interdependence: Measuring Stock Market Comovements," The Journal of Finance, 57(5), 22232261.

Fortin, I., And C. Kusmics (2002): "Tail - Dependence in Stock Return Pairs," The International Journal of Intelligent Systems in Accounting, Finance $\mathcal{G}^{\circ}$ Management, 11(2), 89-107.

Genest, C., K. Ghoudi, and L.-P. Rivest (1995): "A Semiparametric Estimation Procedure of Dependence Parameters in Multivariate Families of Distributions," Biometrika, 82(3), 543-552.

Hu, L. (2006): "Dependence Patterns Across Financial Markets: A Mixed Copula Approach," Applied Financial Economics, 16(10), 717-729.

Hu, W., and A. Kercheval (2007): "The Skewed t Distribution for Portfolio Credit Risk," in Advances in Econometrics, $200 \%$.

Joe, H. (1997): Multivariate Models and Dependence Concepts. Chapman \& Hall. 
Jondeau, E., And M. Rockinger (2001): "Conditional Dependency of Financial Series: The Copula-GARCH Model," Cahier de recherche du groupe HEC 723 / 2001.

Jones, M., And M. FADDy (2003): "A Skew Extension of the t-distribution, With Applications," The Journal of the Royal Statistical Society: Series B, 65(1), 1-329.

Koutmos, G., and G. Boотн (1995): “Asymmetric Volatility Transmissions in International Stock Markets," The Journal of International Money and Finance, 14(6), 747-762.

Longin, F., And B. Solnik (2001): "Extreme Correlation of International Equity Markets," The Journal of Finance, 56(2), 649-676.

McNeil, A., R. Frey, and P. Embrechts (2005): Quantitative Risk Management: Concepts, Techniques and Tools. Princeton University Press.

Menca, J., And E. Sentana (2004): "Estimation and Testing of Dynamic Models with Generalized Hyperbolic Innovations," Working Paper.

Moraux, F., P. Navatte, and C. Villa (1998): "The Predictive Power of the French Market Volatility Index: A Multi Horizons Study," The European Finance Review, 2(3), 303-320.

Nelsen, R. (1999): An Introduction to Copulas. Springer Series in Statistics, New York, 2nd edn.

OAKEs, D. (1994): "Multivariate Survival Distributions," The Journal of Nonparametric Statistics, 3(3\&4), 343-354.

Patton, A. (2006): "Modelling Asymmetric Exchange Rate Dependence," International Economic Review, 47(2), 527-556. 
Prause, K. (1999): "The Generalized Hyperbolic Model: Estimation, Financial Derivatives, and Risk Measures," PhD Thesis, Mathematics Faculty, University of Freiburg.

Shin, J., And T. Louis (1995): "Inferences on the Association Parameter in Copula Models for Bivariate Survival Data," Biometrics, 51(4), 1384-1399.

Simon, D. (2003): "The NASDAQ Volatility Index During and After the Bubble," The Journal of Derivatives, 11(2), 9-24.

Whaley, R. (1993): "Derivatives on Market Volatility: Hedging Tools Long Overdue," The Journal of Derivatives, 1(1), 71-84.

(2000): "The Investor Fear Gauge," The Journal of Portfolio Management, 26(3), 12-17. 


\section{Appendix - The EM Algorithm for the Estima- tion of the Skewed t-Copula}

This section describes the EM algorithm based upon Demarta and McNeil (2005), McNeil, Frey, and Embrechts (2005), and Hu and Kercheval (2007) we apply for the calibration of the skewed t-copula model. Our setting differs from the latter two references in two respects. First, the framework by McNeil, Frey, and Embrechts (2005) is specified to estimate a skewed t-distribution of X. Hu (2006) proposes to directly fit the distribution. In contrast, we explicitly determine the parameter values out of the empirical rank distribution $\hat{F}(\mathbf{u})$ in order to allow for "meta-skewed t-distributions", whose marginals may differ in functional form. Second, in contrast to Demarta and McNeil (2005), the so-called "E step" of our algorithm comprises a numerical maximization of the conditional log-likelihood by altering $\nu$ in order to avoid dependence upon the numerical accuracy of derivative parameters.

Following Demarta and McNeil (2005), we denote $C_{\nu, P, \gamma}^{t}\left(\mathbf{U} \in[0,1]^{d}\right)$ to be the copula of a d-dimensional skewed $t_{\nu, \mathbf{0}, P, \gamma}^{d}(\mathbf{X})$ distribution, with $P$ being a correlation matrix. In general, the skewed t-distribution can be written as

$$
\mathbf{X}=\mu+\gamma W+\sqrt{W} \mathbf{Z}
$$

where $\gamma \in \mathbb{R}^{d} \neq \mathbf{0}, W \sim \operatorname{Ig}(\nu / 2, \nu / 2)$, and independent $\mathbf{Z} \in \mathbb{R}^{d} \sim N(\mathbf{0}, \mathbf{\Sigma}) .{ }^{10}$ The resulting density function is specified by

$$
f(\mathbf{x})=c \frac{K_{\frac{\nu+d}{2}}\left(\sqrt{\left(\nu+(\mathbf{x}-\mu)^{\prime} \mathbf{\Sigma}^{-1}(\mathbf{x}-\mu)\right) \gamma^{\prime} \boldsymbol{\Sigma}^{-1} \gamma}\right) \exp \left((\mathbf{x}-\mu)^{\prime} \boldsymbol{\Sigma}^{-1} \gamma\right)}{\left(\sqrt{\left(\nu+(\mathbf{x}-\mu)^{\prime} \boldsymbol{\Sigma}^{-1}(\mathbf{x}-\mu)\right) \gamma^{\prime} \boldsymbol{\Sigma}^{-1} \gamma}\right)^{-\frac{\nu+d}{2}}\left(1+\frac{(\mathbf{x}-\mu)^{\prime} \boldsymbol{\Sigma}^{-1}(\mathbf{x}-\mu)}{\nu}\right)^{\frac{\nu+d}{d}}}
$$

\footnotetext{
${ }^{10}$ The variable $X$ has the law of an inverse Gamma distribution $\operatorname{Ig}(\alpha, \beta)$ if it follows the density function

$$
f(x)=\frac{\beta^{\alpha} x^{-\alpha-1} e^{-\beta / x}}{\Gamma(\alpha)},
$$

where $x>0, \alpha>0$, and $\beta>0$.
} 
with $K_{\lambda}$ denoting the modified Bessel function of the third kind. $c$ as the normalizing constant is defined as

$$
c=\frac{2^{\frac{2-(\nu+d)}{2}}}{\Gamma\left(\frac{\nu}{2}\right)(\pi \nu)^{\frac{d}{2}}|\Sigma|^{\frac{1}{2}}} .
$$

Defining the vector $\theta=(\mu, \Sigma, \gamma)$ the $\log$-likelihood can be written as

$$
\ln \mathscr{L}\left(\mathbf{X}_{1}, \ldots, \mathbf{X}_{T}, \theta, \nu\right)=\sum_{t=1}^{T} \ln f_{\mathbf{X}}\left(\mathbf{X}_{t}, \theta, \nu\right)
$$

The conditional independence between $\mathbf{X}_{t}$ and $W_{1}, \ldots, W_{T}, \forall t$, leads to

$$
\ln \tilde{\mathscr{L}}\left(\theta, \mathbf{X}_{1}, \ldots, \mathbf{X}_{T}, W_{1}, \ldots, W_{T}\right)=\sum_{t=1}^{T} \ln f_{\mathbf{X} \mid W}\left(\mathbf{X}_{t} \mid W_{t}, \mu, \boldsymbol{\Sigma}, \gamma\right)+\sum_{t=1}^{T} \ln h_{W}\left(W_{t}\right)
$$

This enables us to maximize the two sums separately. The parameters $\mu, \Sigma$, and $\gamma$ are separated from the term controlled by $\nu$. Yet, the distribution of $W_{t}$ is latent. As such, in a first step of the EM algorithm, the "E step", we maximize the expectation of the objective function given the observations $\mathbf{X}_{1}, \ldots, \mathbf{X}_{T}$ and the parameter estimates of algorithm step $k, \theta^{[k]}$

$$
\max _{\nu} E\left(\ln \tilde{\mathscr{L}}\left(\nu, \mathbf{X}_{1}, \ldots, \mathbf{X}_{T}, W_{1}, \ldots, W_{T} \mid \mathbf{X}_{1}, \ldots, \mathbf{X}_{T}, \theta^{[k]}\right)\right.
$$

In the subsequent step, the "M step", the objective function is maximized by altering the parameters $\theta$.

Define $\mathbf{u}_{1}$ and $\mathbf{u}_{2}$ to be the marginal distributions of the bivariate copula $C$. The algorithm can be specified by the following subsequent steps:

- Set the iteration indicator $k=1$. Specify the starting value of $\nu$ as $\nu^{[1]}=30$. In order to determine the "pseudo sample" $\mathbf{X}$, we invert the univariate distributions $\mathbf{u}_{1}$ and $\mathbf{u}_{2}$ by the ordinary symmetric t-distribution $t\left(0,1, \nu^{[1]}\right)$. Set $\mu^{[1]}=\mathbf{0}, \boldsymbol{\Sigma}^{[1]}=\operatorname{Cov}(\mathbf{X})$, and $\gamma^{[1]}$ close to the zero vector to specify a distribution close to the symmetric student t specification.

- For convenience, define

$$
\rho_{t}^{[k]}=\left(\mathbf{X}_{t}-\mu^{[k]}\right)^{\prime}\left(\boldsymbol{\Sigma}^{[k]}\right)^{-1}\left(\mathbf{X}_{t}-\mu^{[k]}\right) .
$$


For the maximization of the augmented likelihood given $W_{t}, t=1, \ldots, T$, we calculate the auxiliary variables

$$
\vartheta_{t}^{[k]}=\left(\frac{\rho_{t}^{[k]}+\nu^{[k]}}{\gamma^{[k] / \Sigma^{[k]-1}} \gamma^{[k]}}\right)^{-\frac{1}{2}} \frac{K_{\frac{\nu+d+2}{2}}\left(\sqrt{\left(\rho_{t}^{[k]}+\nu^{[k]}\right)\left(\gamma^{[k] / \Sigma^{[k]-1}} \gamma^{[k]}\right)}\right)}{K_{\frac{\nu+d}{2}}\left(\sqrt{\left(\rho_{t}^{[k]}+\nu^{[k]}\right)\left(\gamma^{[k] / \Sigma^{[k]-1}} \gamma^{[k]}\right)}\right)}
$$

and

$$
\eta_{t}^{[k]}=\left(\frac{\rho_{t}^{[k]}+\nu^{[k]}}{\gamma^{[k] \prime} \Sigma^{[k]-1} \gamma^{[k]}}\right)^{\frac{1}{2}} \frac{K_{\frac{\nu+d-2}{2}}\left(\sqrt{\left(\rho_{t}^{[k]}+\nu^{[k]}\right)\left(\gamma^{[k] \prime} \boldsymbol{\Sigma}^{[k]-1} \gamma^{[k]}\right)}\right)}{K_{\frac{\nu+d}{2}}\left(\sqrt{\left(\rho_{t}^{[k]}+\nu^{[k]}\right)\left(\gamma^{[k] \prime} \Sigma^{[k]-1} \gamma^{[k]}\right)}\right)} .
$$

In case of the symmetric t-copula we have instead $\theta_{t}^{[k]}=\frac{\nu^{[k]}+d}{\rho_{t}^{[k]}+\nu^{[k]}}, \eta_{t}^{[k]}=$ $\frac{\rho_{t}^{[k]}+\nu^{[k]}}{\nu^{[k]}+d-2}$, and a further auxiliary variable $\xi_{t}^{[k]}=\ln \left(\frac{\rho_{t}^{[k]}+\nu^{[k]}}{2}\right)-\psi\left(\frac{d+\nu^{[k]}}{2}\right)$, where $\psi$ denotes the Digamma function.

- Defining $\bar{\vartheta}^{[k]}=\frac{1}{T} \sum_{t=1}^{T} \vartheta_{t}^{[k]}$ and $\bar{\eta}^{[k]}=\frac{1}{T} \sum_{t=1}^{T} \eta_{t}^{[k]}$, we can update the parameter estimations by

$$
\begin{gathered}
\gamma^{[k+1]}=\frac{\sum_{t=1}^{T} \vartheta_{t}^{[k]}\left(\overline{\mathbf{X}}-\mathbf{X}_{t}\right)}{T\left(\bar{\vartheta}^{[k]} \bar{\eta}^{[k]}-1\right)}, \\
\mu^{[k+1]}=\frac{1 / T \sum_{t=1}^{T} \vartheta_{t}^{[k]} \mathbf{X}_{t}-\gamma^{[k+1]}}{\bar{\vartheta}^{[k]}},
\end{gathered}
$$

and

$$
\boldsymbol{\Sigma}^{[k+1]}=\frac{1}{T} \sum_{t=1}^{T} \vartheta_{t}^{[k]}\left(\mathbf{X}_{t}-\mu^{[k+1]}\right)\left(\mathbf{X}_{t}-\mu^{[k+1]}\right)^{\prime}-\bar{\eta}^{[k]} \gamma^{[k+1]} \gamma^{[k+1] \prime}
$$

- In the subsequent "M step", $\nu^{[k+1]}$ is determined by maximizing the loglikelihood for the skewed t-copula. In the symmetric case, we can numerically solve the equation

$$
-\psi\left(\frac{\nu}{2}\right)+\ln \left(\frac{\nu}{2}\right)+1-\bar{\xi}^{[k]}-\bar{\theta}^{[k]}=0
$$


where $\bar{\xi}^{[k]}=\frac{1}{T} \sum_{t=1}^{T} \xi_{t}^{[k]} \cdot{ }^{11}$

- Set the iteration counter $k=k+1$ and repeat the entire procedure (omitting step 1) until the likelihood estimation converges up to a pre-specified level.

\footnotetext{
${ }^{11}$ In the case of the skewed t-copula, the EM algorithm by Demarta and McNeil (2005), $\nu$ was also determined by numerically solving

$$
-\psi\left(\frac{\nu}{2}\right)+\ln \left(\frac{\nu}{2}\right)+1-\bar{\xi}^{[k]}-\bar{\vartheta}^{[k]}=0
$$

This procedure requires the calculation of

$$
\xi_{t}^{[k]}=\frac{1}{2} \ln \left(\frac{\rho_{t}^{[k]}+\nu^{[k]}}{\gamma^{[k] / \boldsymbol{\Sigma}^{[k]-1} \gamma^{[k]}}}\right)+\frac{\left.\frac{\partial K_{-\frac{\nu+d}{2}+\alpha}\left(\sqrt{\left(\rho_{t}^{[k]}+\nu_{t}^{[k]}\right)\left(\gamma^{\left.[k] \prime \boldsymbol{\Sigma}^{[k]-1} \gamma^{[k]}\right)}\right)}\right.}{\partial \alpha}\right|_{\alpha=0}}{K_{\frac{\nu+d}{2}}\left(\sqrt{\left(\rho_{t}^{[k]}+\nu_{t}^{[k]}\right)\left(\gamma^{[k] \prime} \boldsymbol{\Sigma}^{[k]-1} \gamma^{[k]}\right)}\right)}
$$

and $\bar{\xi}_{t}^{[k]}=\frac{1}{T} \sum_{t=1}^{T} \xi_{t}^{[k]}$, which depend upon the specification of $\partial \alpha$. In order to avoid this dependence, we decide to directly determine $\nu$ by a gradient method, although this requires more computational effort.
} 


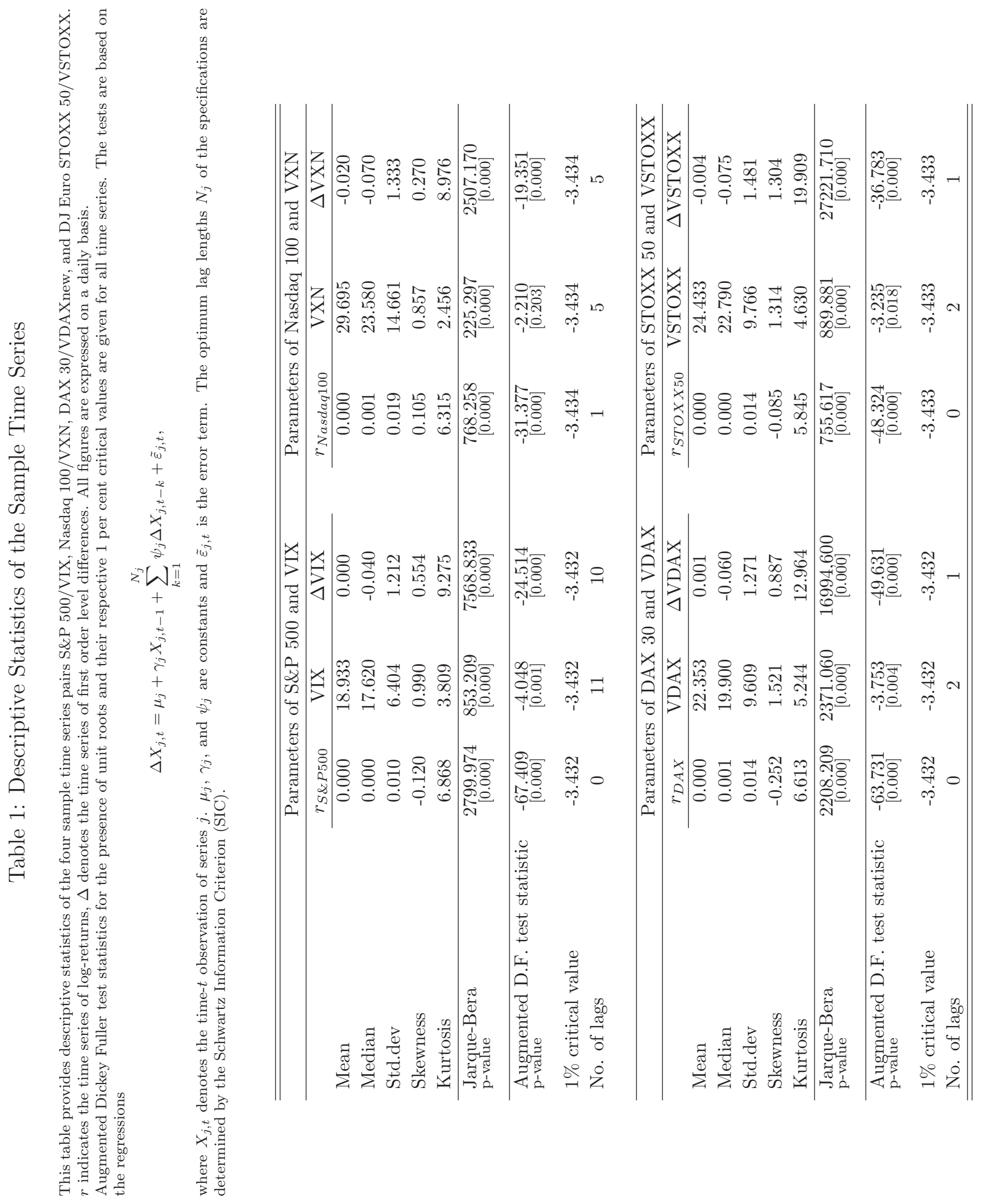


Table 2: Parameter Estimates for the Univariate Marginal Distributions This table illustrates the parameter estimates of the marginal distributions. All univariate processes $j$ are specified as auto-regressive $\operatorname{GARCH}(1,1)$ models which can be written as

$$
\begin{aligned}
& x_{j, t}=\alpha+\sum_{k=1}^{K_{j}} \phi_{k} x_{j, t-k}+\sigma_{j, t} \tilde{\varepsilon}_{j, t} \\
& \sigma_{j, t}^{2}=\omega_{j}+\beta_{j} \varepsilon_{j, t-1}^{2}+\gamma \sigma_{j, t-1}^{2},
\end{aligned}
$$

where $\tilde{\varepsilon}_{j, t}$ follows the law of a student t-distribution with $\nu_{j}$ degrees of freedom, i.e. $\varepsilon \sim t_{\nu_{j}}(0,1) . K_{j}$ is determined by optimizing the Schwarz Information Criterion (BIC). Corresponding p values are stated in brackets. emptyempty

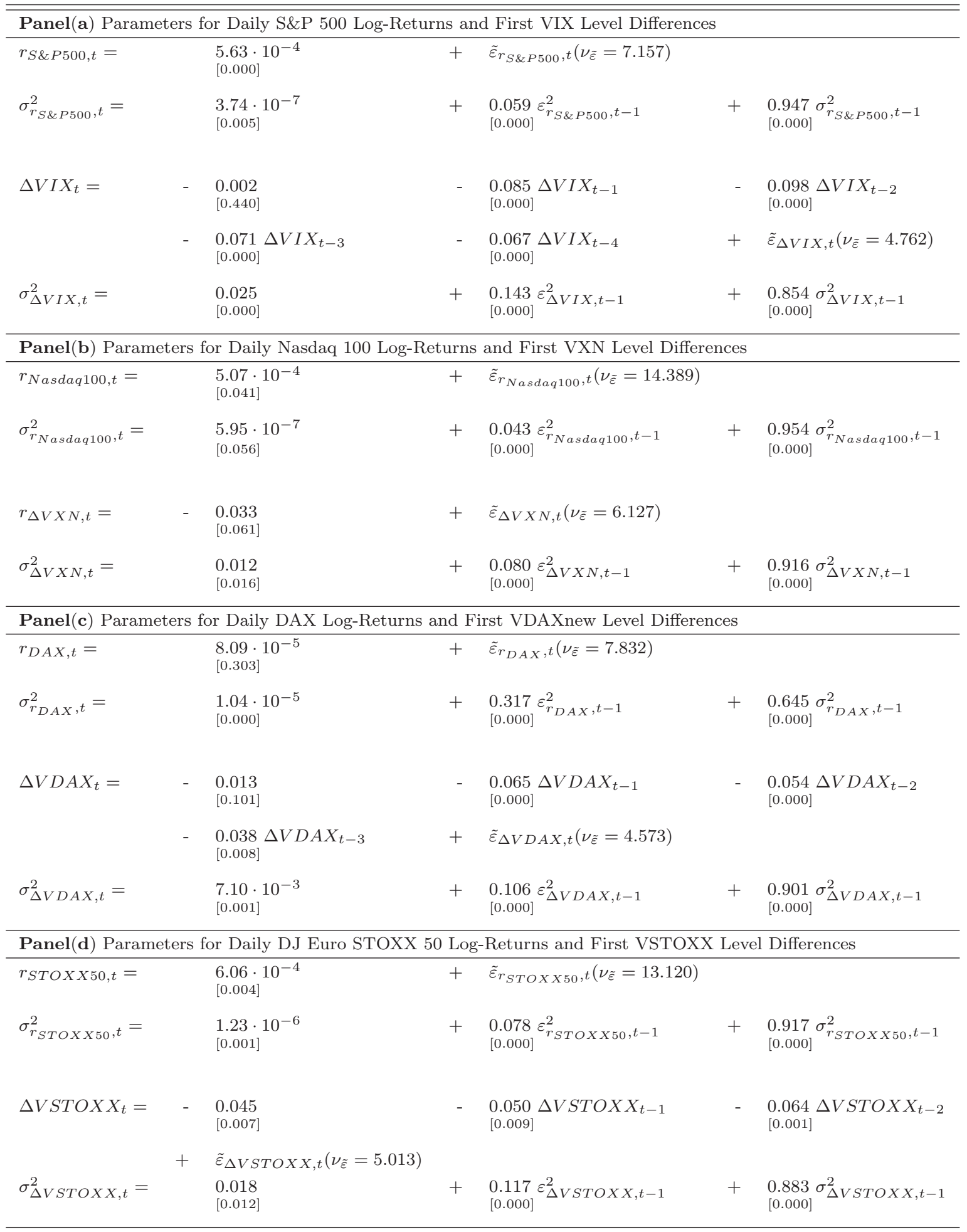


Table 3: Empirical Descriptions of the Dependence Pattern between the Time Series of Equity Index Log-Returns and Daily Changes of Implied Volatility Indices

This table outlines "empirical joint conditional quantile exceedance probabilities" for daily equity index logreturn time series and daily implied volatility index level differences. They are defined as

$$
\frac{\operatorname{Prob}\left(r_{\text {EquityIndex }} \leq u, \Delta \text { VolatilityIndex } \geq 1-u\right)}{u},
$$

for $u=0.05,0.1$, and 0.2. Analogously,

$$
\frac{\operatorname{Prob}\left(r_{\text {EquityIndex }} \geq \bar{u}, \Delta \text { VolatilityIndex } \leq 1-\bar{u}\right)}{1-\bar{u}},
$$

where $\bar{u}=0.8,0.9$, and 0.95 . The values in brackets give the magnitude that upper-left tail probabilities outrange their corresponding lower-right tail counterparts. Values are calculated for the S\&P 500/VIX, the

\begin{tabular}{|c|c|c|c|c|c|c|}
\hline & \multicolumn{3}{|c|}{ Upper-Left Tail $u$} & \multicolumn{3}{|c|}{ Lower-Right Tail $\bar{u}$} \\
\hline & 0.05 & 0.1 & 0.2 & 0.8 & 0.9 & 0.95 \\
\hline$u\left(r_{S \& P 500}\right) / u(\Delta V I X)$ & $\begin{array}{c}0.608 \\
{[131.25 \%]}\end{array}$ & $\begin{array}{c}0.577 \\
{[136.39 \%]}\end{array}$ & $\begin{array}{c}0.319 \\
{[34.69 \%]}\end{array}$ & 0.451 & 0.244 & 0.138 \\
\hline$u\left(r_{N \text { asdaq } 100}\right) / u(\Delta V X N)$ & $\begin{array}{c}0.236 \\
{[23.94 \%]}\end{array}$ & $\begin{array}{c}0.418 \\
{[48.09 \%]}\end{array}$ & $\begin{array}{c}0.566 \\
{[21.39 \%]}\end{array}$ & 0.466 & 0.282 & 0.191 \\
\hline$u\left(r_{D A X}\right) / u(\Delta V D A X n e w)$ & $\begin{array}{c}0.322 \\
{[108.67 \%]}\end{array}$ & $\begin{array}{c}0.406 \\
{[131.30 \%]}\end{array}$ & $\begin{array}{c}0.486 \\
{[39.56 \%]}\end{array}$ & 0.348 & 0.176 & 0.155 \\
\hline$u\left(r_{S T O X X 50}\right) / u(\Delta V S T O X X)$ & $\begin{array}{c}0.622 \\
{[709.36 \%]}\end{array}$ & $\begin{array}{c}0.527 \\
{[139.86 \%]}\end{array}$ & $\begin{array}{c}0.620 \\
{[31.46 \%]}\end{array}$ & 0.472 & 0.220 & 0.077 \\
\hline
\end{tabular}
Nasdaq 100/VXN, the DAX/VDAXnew, and the DJ Euro STOXX 50/VSTOXX time series pairs. 


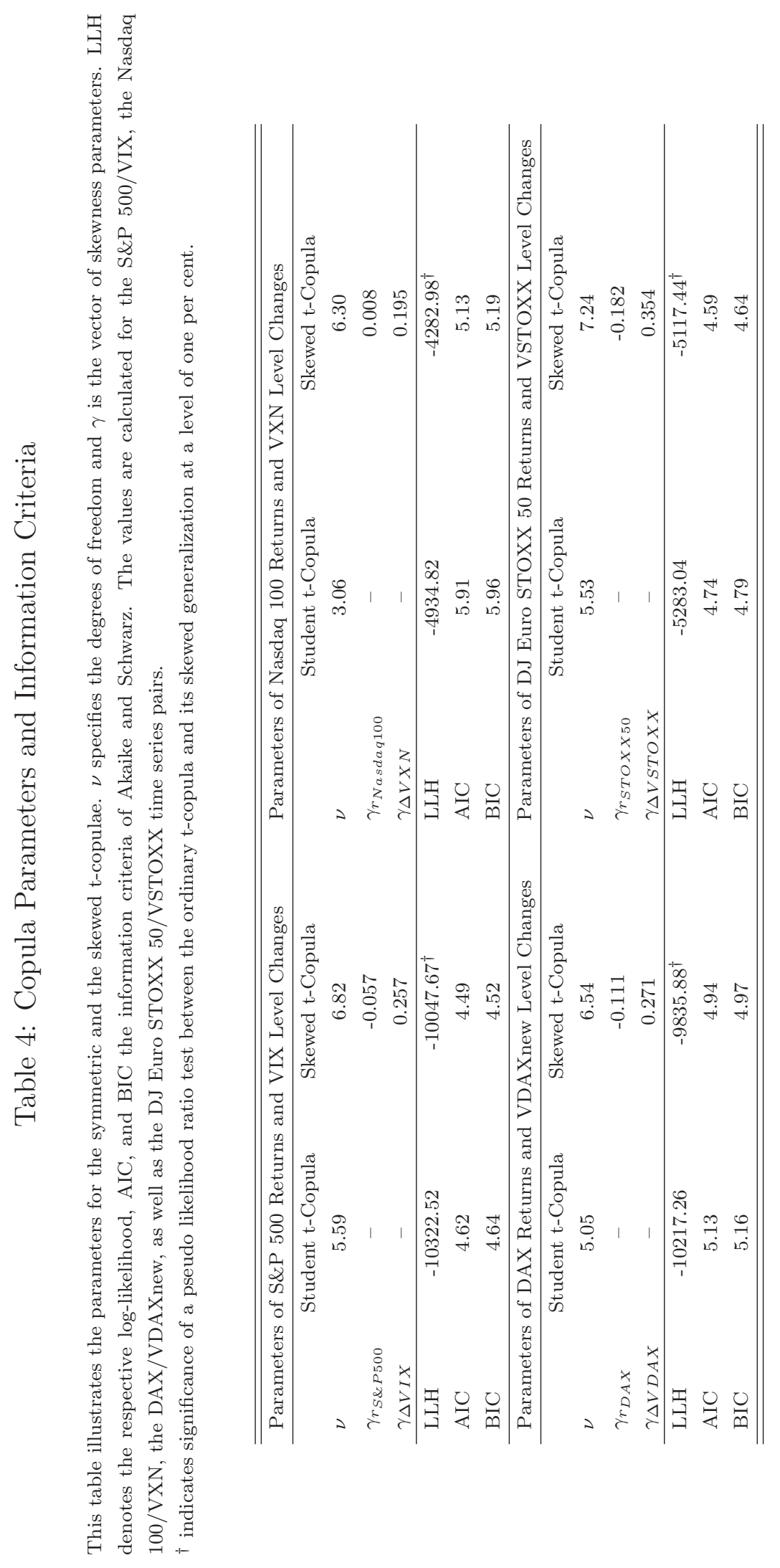


Figure 1: Scatter Plot of Daily Equity Index Log-Returns and Changes in the Corresponding Volatility Index Levels

Figure (a) illustrates a scatter plot of daily VIX index level changes and S\&P 500 log-returns for the period from January $3^{\text {rd }}$, 1990, until September $29^{\text {th }}$, 2007. After the exclusion of non-trading days, the sample comprises a total of 4474 observations for each time series.

Figure (b) shows the respective plot for the Nasdaq 100/VXN time series. The sample covers the period from February $5^{\text {th }}, 2001$, until September $29^{t h}, 2007$, with a total of 1671 observations.

Figure (c) gives the scatter plot of the DAX/VDAXnew time series combination for the January $3^{\text {rd }}, 1992$, to September $29^{t h}, 2007$, period with a total of 3982 data points for each time series after the exclusion of non-trading days.

Figure (d) outlines the scatter plot of daily DJ Euro STOXX 50 returns versus the corresponding level changes of the VSTOXX volatility index. Our sample covers the period from January $5^{t h}$, 1999, to September $29^{\text {th }}$, 2007, resulting in 2232 data point combinations.

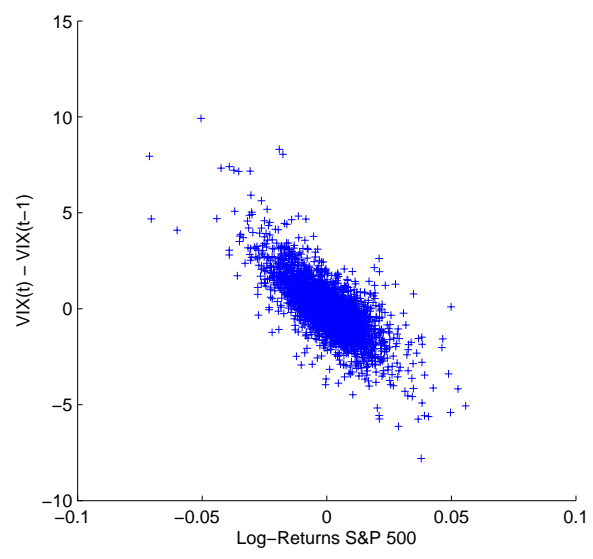

(a) S\&P 500 Log-Returns and VIX Level Changes

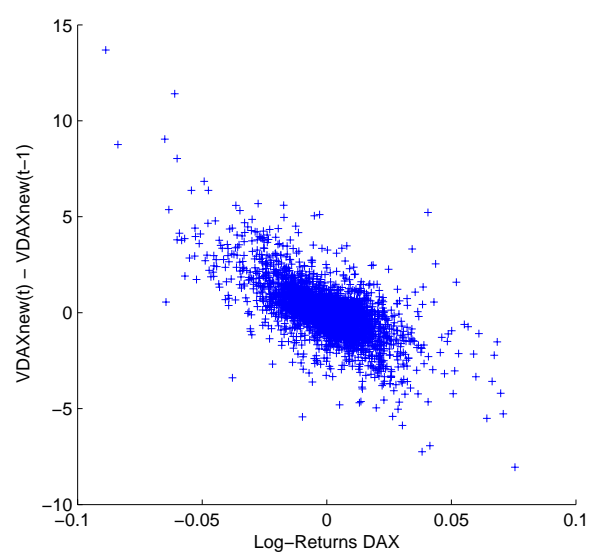

(c) DAX Log-Returns and VDAXnew Level Changes

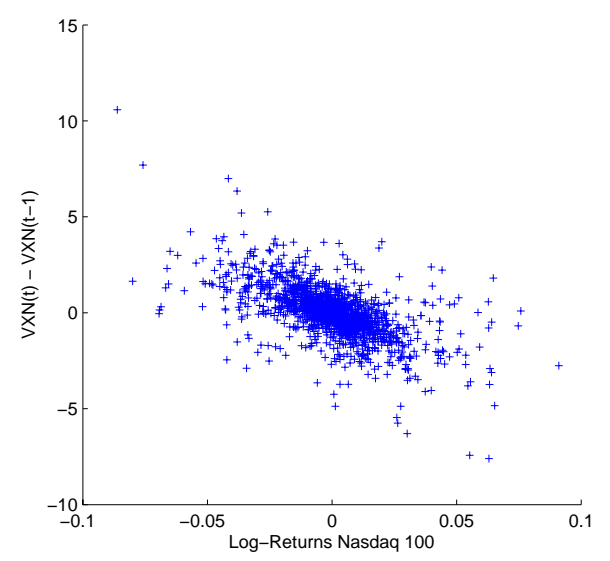

(b) Nasdaq 100 Log-Returns and VXN Level Changes

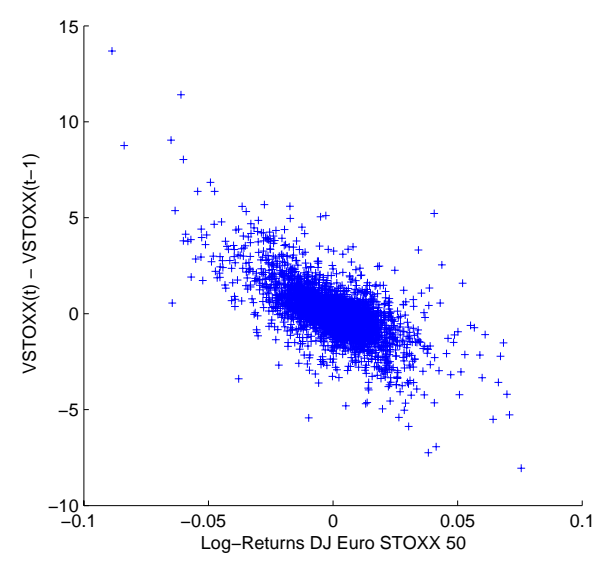

(d) DJ Euro STOXX 50 Log-Returns and VSTOXX Level Changes 


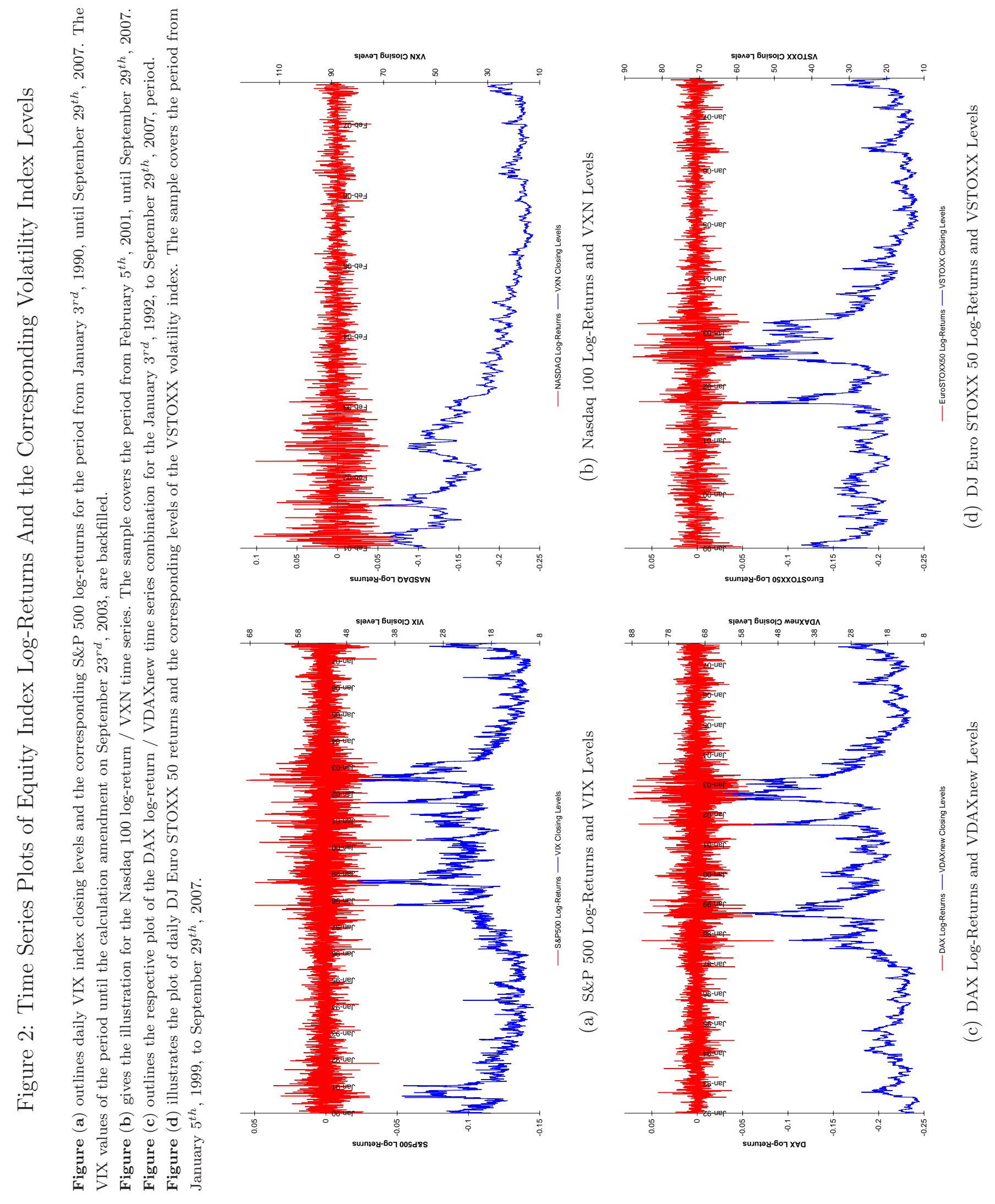


Figure 3: Correlation Structure between Volatility Index Level Changes and Equity Index Log-Return Exceedances

This figure illustrates the correlation structure of volatility index level differences and the corresponding log-return exceedances. All calculations are based on subsamples including all observations $\mathbf{X}_{\vartheta}$ with $r_{\text {EquityIndex }}\left(\mathbf{X}_{\vartheta}\right) \in(-\infty, \vartheta], \forall \vartheta \leq 0$ and $r_{\text {EquityIndex }}\left(\mathbf{X}_{\vartheta}\right) \in[\vartheta, \infty), \forall \vartheta>0$, respectively, where $r$ defines the equity index log-return and $\vartheta$ the respective threshold level. Figures(a) to (d) show the correlation structures for the S\&P 500/VIX (a), the Nasdaq 100/VXN (b), DAX/VDAXnew (c), and DJ Euro STOXX 50/VSTOXX (d) time series combinations.

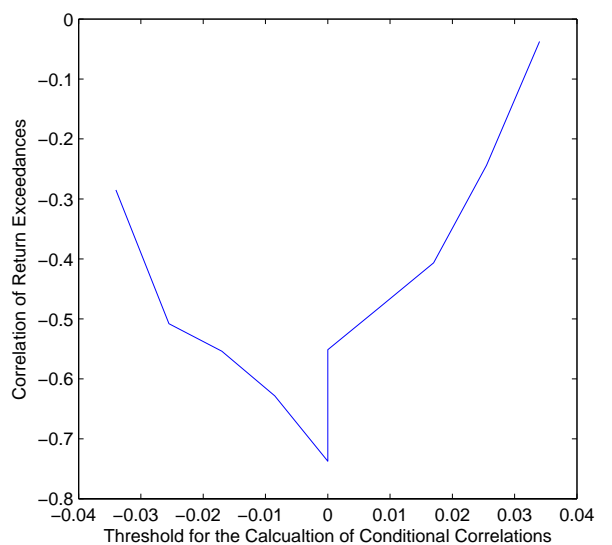

(a) Conditional Correlation Figures between S\&P 500 Log-Returns and VIX Level Changes

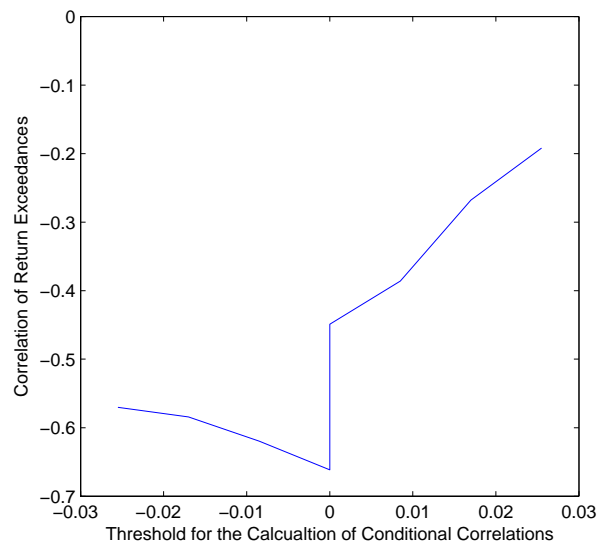

(c) Conditional Correlation Figures between DAX Log-Returns and VDAXnew Level Changes

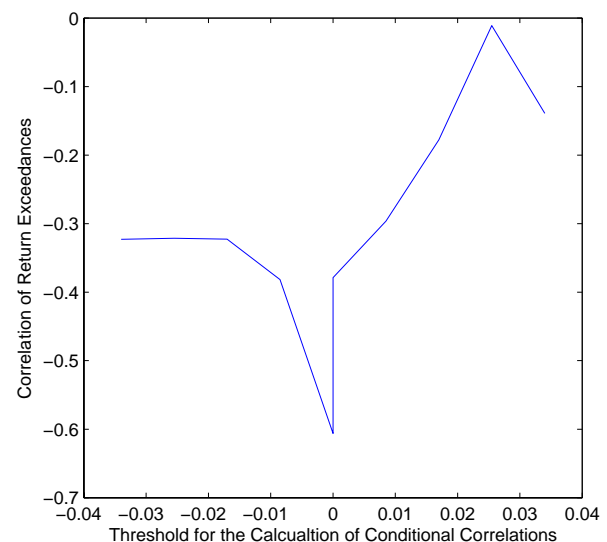

(b) Conditional Correlation Figures between Nasdaq 100 Log-Returns and VXN Level Changes

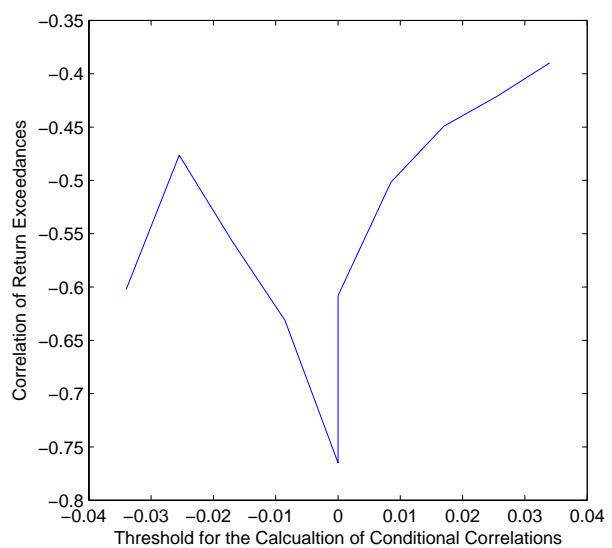

(d) Conditional Correlation Figures between DJ Euro STOXX 50 Log-Returns and VSTOXX Level Changes 
Figure 4: Empirical Quantile Distribution of Daily Volatility Index Level Changes and Equity Index Log-Returns

These figures illustrate daily empirical quantile distributions of the marginal distribution's innovations mapped into the unit square. Each marginal time series $j$ is modeled as an auto-regressive $\operatorname{GARCH}(1,1)$ process, specified as

$$
\begin{aligned}
& x_{j, t}=\alpha+\sum_{k=1}^{K_{j}} \phi_{k} x_{j, t-k}+\sigma_{j, t} \tilde{\varepsilon}_{j, t} \\
& \sigma_{j, t}^{2}=\omega_{j}+\beta_{j} \varepsilon_{j, t-1}^{2}+\gamma \sigma_{j, t-1}^{2},
\end{aligned}
$$

where $\tilde{\varepsilon}_{j, t}$ follows a student t-distribution with $\nu_{j}$ degrees of freedom. $K_{j}$ is determined by the minimization of the Schwarz information Criterion (BIC) for each time series $j$. Quantiles are calculated using the probability integral transform.

Figures (a) to (d) illustrate the empirical quantile distributions for the S\&P 500/VIX (a), the Nasdaq 100/VXN (b), DAX/VDAXnew (c), and DJ Euro STOXX 50/VSTOXX (d) time series combinations.

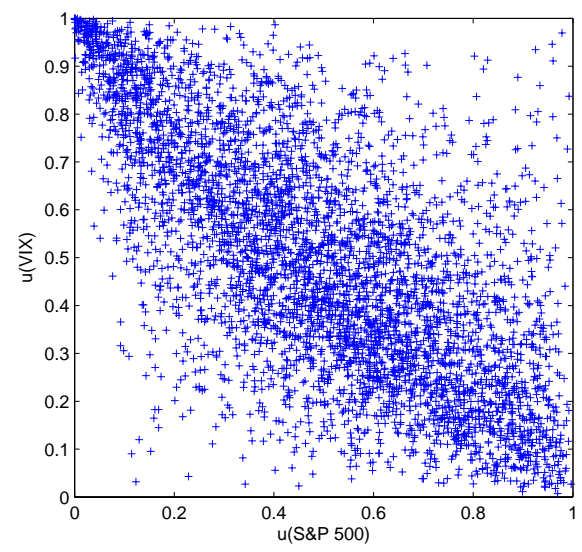

(a) Scatter Plot of $u\left(r_{S \& P 500}\right)$ and $u(\Delta V I X)$

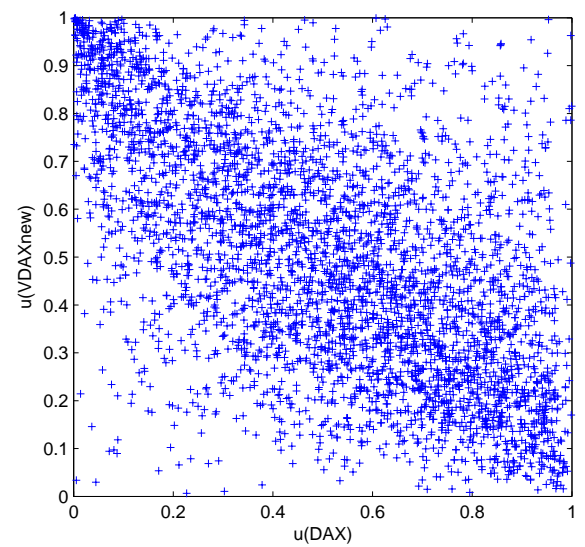

(c) Scatter Plot of $u\left(r_{D A X}\right)$ and $u(\triangle V D A X$ new $)$

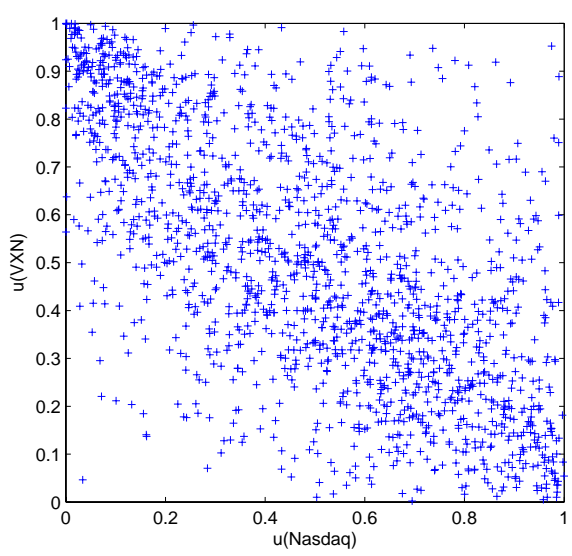

(b) Scatter Plot of $u\left(r_{N a s d a q 100}\right)$ and $u(\Delta V X N)$

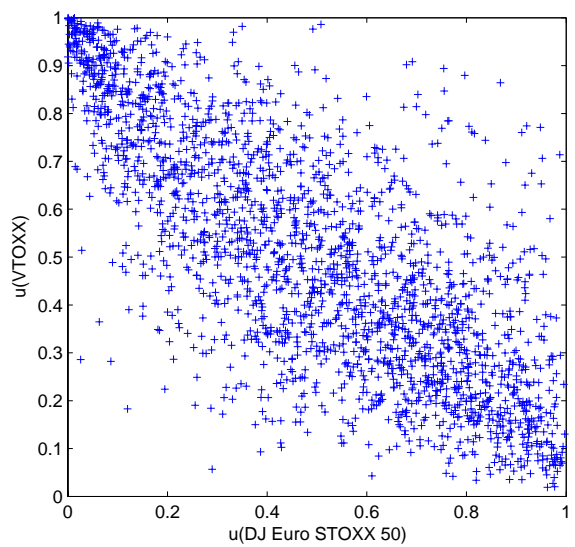

(d) Scatter Plot of $u\left(r_{D J E u r o S t o x x 50}\right)$ and $u(\triangle V S T O X X)$ 